\begin{tabular}{|c|l|}
\hline Title & Porous microfibers and microhoneycombs synthesized by ice templating \\
\hline Author(s) & Mukai, Shin R.; Nishihara, Hirotomo; Tamon, Hajime \\
\hline Citation & $\begin{array}{l}\text { Catalysis Surveys from A sia, 10(3-4), 161-171 } \\
\text { https://doi.org/10.1007/310563-006-9015-8 }\end{array}$ \\
\hline Issue Date & 2006-12 \\
\hline Doc URL & http://hdl.handle.net/2115/17122 \\
\hline Rights & The original publication is available at www.springerlink.com \\
\hline Type & article (author version) \\
\hline File Information & CSFA 10-3-4.pdf \\
\hline
\end{tabular}

Instructions for use 


\title{
Porous Microfibers and Microhoneycombs by Ice Templating
}

\author{
${ }^{\mathrm{a}}$ Shin R. Mukai, ${ }^{\mathrm{b}}$ Hirotomo Nishihara and ${ }^{\mathrm{c}}$ Hajime Tamon \\ ${ }^{a}$ Graduate School of Engineering, Hokkaido University, Sapporo, 060-8628, Japan \\ ${ }^{\mathrm{b}}$ Institute of Multidisciplinary Research for Advanced Materials, Tohoku University, Sendai, 980-8577, Japan \\ ${ }^{\mathrm{c}}$ Graduate School of Engineering, Kyoto University, Kyoto, 615-8510, Japan
}

\begin{abstract}
Recently we introduced the "ice templating" method, a new method which allows the synthesis of nanoporous materials with unique morphology, such as microfibers and microhoneycombs. In this method, materials are synthesized by freezing their parent hydrosols or hydrogels unidirectionally. Ice crystals which grow within the precursor during freezing act as the template. Therefore, the template can be easily removed through simple thawing and drying, which is a unique and beneficial feature of this method.
\end{abstract}

This paper will first describe the outline of this new method and next, the details about methods to control the dimensions of the materials obtained through it. Comments about the range of applicability of this method will also be provided.

Keywords: Porous Materials, Sol-Gel, Freeze Gelation, Unidirectional Freezing, Microfiber, Microhoneycomb

\section{INTRODUCTION}

Porous materials are widely used as catalysts and adsorbents, and recently they have become the key materials in energy storage devices, such as lithium ion batteries and super capacitors. Micropores and smaller mesopores mainly provide the unique functions of porous materials, but substances which can receive benefits from such functions can gain access to the micropores and smaller mesopores only by passing through the macropores or larger mesopores of the material. Moreover, substances which have received benefits must pass through such larger pores to come out of the material. Such transport processes are extremely important, and in certain cases, they determine the overall performance of the 
material itself.

Considering these facts, it can be said that one form of an ideal porous material is a material with a hierarchical pore system of micro-, meso- and macropores, which ratios among these 3 types of pores are tunable. If such a material can be obtained, for example, the energy density of a material for energy storage might be maximized by tuning the pore system to have a tremendous amount of micropores, with the minimum amount of mesopores which provide pathways to the micropores, and extremely high selectivities may be achieved by tuning the pore system of a catalyst to have a significant amount of short micropores which are all directly connected to macropores. Rapid and efficient separation might be achieved by tuning the pore system to have moderate amounts of macropores and mesopores. There are numerous reports which deal with the designing of meso- and micropores, so the designing of macropores is thought to be the key to obtain such materials.

This is one reason why porous materials with ordered macropores, have recently drawn much attention. Materials with ordered macropores, can be synthesized through various methods, but a majority of them are synthesized using a "sacrificial" template [1]. First, a template with a desired structure is prepared, and then the skeletal material is formed around the template. Next, the template is removed by physical and/or chemical treatment. Typical templates are phase-separated polymers [2-6], colloidal crystals [7-10] and microemulsions $[11,12]$. The voids which are formed after the removal of the template become the macropores. This indicates that if the skeletal material is originally microporous or mesoporous, a hierarchical pore system in which micropores and mesopores are directly connected to macropores can be constructed.

Unique materials with ordered macropores can be obtained through such templating methods, but these methods have common drawbacks. First, the templates used for the synthesis of them are generally extremely expensive. Such templates have to be removed to form macropores, but the removal process of such templates generally requires severe conditions such as extremely high or low $\mathrm{pH}$ and/or high temperatures. We thought that these problems can be overcome by using ice crystals as the template. Ice crystals are extremely inexpensive, and can be removed by simply thawing and drying the material. As can be seen, for example, during the synthesis of frozen foods, it is quite simple to generate numerous ice crystals with fairly uniform sizes just by simple freezing. Moreover, the emission of toxic wastes during synthesis can be avoided if ice crystals are used as the template. Such an idea occurred to us when we 
came across two industrial techniques which exploit freezing, the freeze gelation method [13-15], and the unidirectional freezing method [16-18].

Freeze gelation is a technique which uses freeze concentration to accelerate sol-gel polycondensation. When a sol is frozen, pure ice crystals, which sizes are fairly uniform, are randomly formed within it, resulting in the concentration of the sol between the growing ice crystals. This concentration leads to the acceleration of sol-gel polycondensation and the sol readily transfers to a gel. This concentration effect is extremely significant. For example, a sol which is extremely stable at room temperature can be easily transformed to a gel by freezing. Microstructures which maintain the unique morphology induced during freezing can be obtained by thawing and drying the frozen material.

In unidirectional freezing, a hydrogel of a metal oxide is frozen unidirectionally. Such freezing can be conducted, for example, by dipping the material into a cold bath which temperature is maintained below the freezing point of the material. During unidirectional freezing, an array of needle-shaped ice crystals appears within the hydrogel and elongates in the direction of freezing. This causes the partitioning and condensation of the gel between the growing ice crystals. Gel fibers with polygonal cross sections can be obtained by thawing and drying materials frozen this way.

The unidirectional freezing method was originally developed to obtain long and strong fibers, so this method was solely applied to thoroughly aged hydrogels which structures were hard and firm. However, as is proven in freeze gelation, freezing accelerates sol-gel polycondensation, therefore we assumed that the unidirectional freezing method can also be applied to soft hydrogels and even to hydrosols. By widening the range of application, gels in forms other than polygonal fibers can be expected. Based on this concept, the "ice templating" method was developed [19-25].

This paper will first describe the outline of this new method and next, the details about methods to control $\mu \mathrm{m}$ scale dimensions (fiber diameter and microhoneycomb channel size) and $\mathrm{nm}$ scale dimensions (micropore and mesopore size) of the materials obtained through it. Comments about the range of applicability of this method will also be provided.

\section{ICE TEMPLATING METHOD}

Silica was selected as the model substance to confirm the applicability of unidirectional freezing to soft hydrogels and hydrosols $[19,20]$. Silica can be prepared through a wide variety of methods using 
various sources. Here we prepared silica from a sodium silicate solution through sol-gel polycondensation. First, a commercial sodium silicate solution was diluted with pure water to a desired Si concentration. Next, the $\mathrm{pH}$ of the solution was adjusted using an ion exchange resin. Then, the resulting solution was poured into a polypropylene tube. The tube was maintained at a certain temperature and the solution in it was aged for a certain period. Normally, the solution in the tube will become viscous during this aging process, and after a certain time elapses, the solution will transform to a hydrogel. The firmness of the silica precursor was adjusted by controlling the time allowed for it to age before applying unidirectional freezing.

We unidirectionally froze solutions aged for different times by dipping the tubes which include them into a cold bath at a constant rate. After the samples were completely frozen, they were aged for a certain period and thawed. Finally, the thawed samples were dried. The usage of freeze drying in this drying process enables the further development of nanopores in the resulting material.

When thoroughly aged hydrogels were froze unidirectionally, as expected, microfibers with polygonal cross sections were obtained (Fig. 1 (d)). However, we found that silica with 3 other types of morphology can be obtained by adjusting the firmness of the precursor before applying unidirectional freezing. The unidirectional freezing of sols with low viscosity gave lamella sheets (Fig. 1 (a)), and as the precursor sol became viscous, flat fibers appeared (Fig. 1 (b)). Sols which are about to transform to a gel, and freshly gelled hydrogels gave microhoneycombs (Fig. 1 (c)). As all 4 types of morphology are obtained using ice crystals as the template, we named this method the ice templating method. Among the 4 types of morphology, microhoneycombs and polygonal fibers are expected to have many practical applications.

Figure 2 shows a confocal laser micrograph of typical silica polygonal fibers obtained through ice templating. It can be noticed that the fibers are translucent, and have $\mu \mathrm{m}$-sized diameters. Therefore it is assumed that the ice crystals formed around them during synthesis are straight, and also have $\mu \mathrm{m}$-sized polygonal cross sections. Through nitrogen adsorption experiments, it was confirmed that such fibers have developed nanopores within their structure (Fig. 3). Therefore, if a bundle of such fibers is packed into a column, $\mu \mathrm{m}$-sized pathways will be formed between the aligned fibers, so this column can be considered as a material with a hierarchical pore system, in which nanopores are directly connected to $\mu \mathrm{m}$-sized macropores.

The overall shape of a microhoneycomb obtained through ice templating depends on the shape of the 
vessel used for synthesis. We typically use polypropylene tubes, so cylindrical monolithic honeycombs, as shown in Fig. 4 (a) are obtained. By observing the micrographs of the cross section (Fig. 4 (b), (c)) and vertical section (Fig. 4 (d)) of the silica microhoneycomb, it can be noticed that the cells of the microhoneycomb are $\mu \mathrm{m}$-sized straight channels. As such channels are formed through the removal of ice crystals which were formed during unidirectional freezing, this result indicates that the ice crystals are straight and have $\mu \mathrm{m}$-sized polygonal cross sections. A high magnification micrograph of the honeycomb wall (Fig. 5 (a)) tells us that the walls of the cells are formed by nanoparticles, and have developed nm-sized pores within them. These facts were also confirmed through adsorption experiments (Fig. 5 (b)). Therefore such microhoneycombs are also materials equipped with a hierarchical pore system. Interestingly, it was found that both microhoneycombs having microporous walls (SMH-1), and those having mesoporous walls (SMH-2) can be synthesized by adjusting preparation conditions, such as the $\mathrm{pH}$ of the starting solution.

\section{STRUCTURE CONTROLLING}

As we found that materials with unique hierarchical pore systems can be obtained through ice templating, next we attempted the precise controlling of the hierarchical pore system [23].

The first step in structure controlling is morphology controlling. As mentioned above, in the ice templating method, the morphology of the sample depends on the firmness of its precursor. The firmness of a hydrogel is governed by two factors, the intrinsic tendency of the gel to harden, and the extent of the sol-gel reaction. The first factor can be controlled by preparation conditions such as the Si concentration and $\mathrm{pH}$ of the starting solution in the case of silica, and the intrinsic tendency of the gel to harden can be judged by the gelation time. The second factor can be adjusted by simply controlling the reaction time. Therefore it is assumed that precise morphology controlling can be conducted by adjusting these 2 factors.

The next step in structure controlling is the controlling of $\mu \mathrm{m}$ scale structures, or in other words the controlling of the channel size of microhoneycombs, and the diameter of microfibers. Mahler et al. reported that the freezing of hydrogels is a similar process to the solidification of eutectic alloys [16]. It is known that when a molten eutectic alloy is solidified unidirectionally and a cellular structure appears, the intercellular spacing can be expressed as a function of $(G R)^{-1}$, where $G$ is the temperature gradient at the liquid-solid interface and $R$ the solidification rate [26]. In ice templating, $R$ can be taken as the freezing 
rate $v_{\mathrm{f}}$, and it is natural to assume that $G$ is proportional to the difference between room temperature $T_{\mathrm{r}}$ and freezing temperature $T_{\mathrm{f}}$. Therefore, it is assumed that the sizes of $\mu \mathrm{m}$ scale structures will be proportional to $\left\{v_{\mathrm{f}}\left(T_{\mathrm{r}}-T_{\mathrm{f}}\right)\right\}^{-1}$.

In order to confirm this relationship, we actually produced silica fibers and microhoneycombs under various freezing conditions and plotted their diameters and channel sizes against the freezing parameter, $\left\{v_{\mathrm{f}}\right.$ $\left.\left(T_{\mathrm{r}}-T_{\mathrm{f}}\right)\right\}^{-1}$. Figure 6 shows the results of silica microhoneycombs. In can be noticed that as expected, the channel size of microhoneycombs can be expressed as a function of $\left\{v_{\mathrm{f}}\left(T_{\mathrm{r}}-T_{\mathrm{f}}\right)\right\}^{-1}$. The same trend was observed in the case of silica microfibers. This indicates that in ice templating, the sizes of $\mu \mathrm{m}$ scale structures can be easily controlled by simply adjusting freezing conditions. This is an extremely advantageous feature, since the size of macropores in such materials is extremely important, as it significantly affects the mass transfer abilities of the material, particularly the pressure drop which occurs when fluids are passed through them.

The final step in structure controlling is the controlling of nm scale structures, namely the sizes of mesopores and micropores. Although the apparent density of silica microhoneycombs obtained through ice templating is around $0.1 \mathrm{~g} / \mathrm{cm}^{3}$, which is an extremely low value close to silica aerogels, the density of the walls which form it is around $1.2 \mathrm{~g} / \mathrm{cm}^{3}$, a value quite similar to those of conventional silica gels. Moreover, we have confirmed through various structural analysis methods that the walls of silica microhoneycombs have nanostructures identical to conventional silica gels. Therefore, it is expected that conventional methods for controlling the micro- and mesoporosity of silica gels can also be used in the ice templating method.

We showed that it is possible to change the micro- and mesoporosity of materials obtained through ice templating, by adjusting the $\mathrm{pH}$ of their parent sols [19]. However, the $\mathrm{pH}$ of silica sols significantly affects the time required for it to transfer to a hydrogel, and the firmness of the resulting silica hydrogel. For example, when the $\mathrm{pH}$ is around 5, silica sols rapidly transform to gels, and they quickly become too firm to be transformed into a honeycomb structure. This indicates that there is a $\mathrm{pH}$ region in which it is impossible to obtain a microhoneycomb structure. Therefore in ice templating, the controlling of the $\mathrm{pH}$ of the parent sol is not an effective method to control the micro- and mesoporosity of the resulting material.

It is known that when silica gels with micro- and mesopores are immersed into a basic solution, smaller nanoparticles which compose the silica gel matrix dissolve and reprecipitate on the surface of larger 
nanoparticles, a phenomenon often referred to as "Ostwald ripening." This leads to an increase in the average size of the pores. Therefore, it is expected that the sizes of nanopores can be controlled in a wide range by adopting this hydrothermal treatment method. Moreover, as $\mu \mathrm{m}$ scale structures such as fiber diameter and microhoneycomb channel size are already determined at the freezing stage of ice templating, it is expected that the micro- and mesoporosity of the samples can be controlled independently from their macroporosity if this hydrothermal treatment method is employed.

To verify this, silica samples were synthesized through unidirectional freezing and after thawing them, they were immersed into $\mathrm{NH}_{3}$ solutions of different concentrations $C_{\mathrm{N}}$ and temperatures $T_{\mathrm{ht}}$, and were treated for 5 days. The nitrogen adsorption isotherms of typical samples, and mesopore size distributions derived from them are respectively compared in Figs. 7 and 8. Sample (a), which was not hydrothermally treated, shows a type I isotherm, which indicates that it is microporous. However, the treated samples show type IV isotherms, which indicates that they have developed mesopores. It was found that the sizes of micro- and mesopores in the walls which form the microhoneycombs can be controlled in a wide range of 2 to $45 \mathrm{~nm}$ by adjusting treatment conditions. It was also found that the microporosity of the samples tend to decrease with the increase in $T_{\mathrm{ht}}$. On the other hand, the sizes of mesopores increase, and the mesopore size distribution tends to broaden with the increase in $T_{\mathrm{ht}}$. The effects of $C_{\mathrm{N}}$ were much more different than those of $T_{\mathrm{ht}}$. Only the sizes of mesopores changed when $C_{\mathrm{N}}$ was increased. Therefore, it can be said that by increasing $C_{\mathrm{N}}$, it is possible to increase the sizes of mesopores without hardly changing the total volume of mesopores and the sharpness of their size distribution. These results are consistent with results previously reported [27].

From the results shown above, it can be concluded that a method to precisely control the morphology and hierarchical pore structure of materials obtained through ice templating has been established. By using this method, the morphology and the pore system of the resulting material can be tuned to make best use of the properties of the material in various processes.

\section{RANGE OF APPLICABILITY}

We also conducted studies to clarify the range of applicability of the ice templating method. In principle, the ice templating method can be applied to materials which can be obtained through sol-gel polycondensation. As silica is a typical inorganic material, next we verified the possibility to apply the ice 
templating method to organic gels $[21,24]$.

Resorcinol formaldehyde (RF) gels were selected to represent this category, due to the similarity of its structure and preparation method to those of silica gels $[28,29]$. RF hydrogels were prepared through the sol-gel polycondensation of resorcinol with formaldehyde using sodium carbonate as the catalyst and pure water as the diluent. First, the 4 ingredients were mixed, and after a homogeneous solution was obtained, the solution was cast into glass molds. The samples were maintained at a constant temperature, and after sol-gel transition occurred, the resulting RF hydrogels were aged for certain periods. Then the samples were thoroughly washed with pure water, and were frozen unidirectionally and thawed. A part of the resulting samples were treated hydrothermally in order to reinforce the network structure of the hydrogels and avoid the contraction of the nanopores formed within it. Finally, the samples were freeze-dried.

It was found that, like other gels, microhoneycombs and microfibers of RF gels can also be obtained using the ice templating method. Figures 9 (a) and 9 (b) show the cross-sections of a typical RF microhoneycomb. It can be noticed that the microhoneycombs have straight $\mu \mathrm{m}$-sized channels. It was confirmed that the walls of the microhoneycomb are composed of nanosized particles, like in typical RF gels. Figures 9 (c) and 9 (d) respectively show a photograph of microfibers, and a micrograph of the cross section of a single microfiber. These microfibers were also composed of nanosized particles.

Figures 10 and 11 respectively show nitrogen adsorption-desorption isotherms of typical RF microhoneycombs, and the mesopore size distributions derived from the isotherms. Only slight pore development was observed in sample MH-0, a microhoneycomb prepared without hydrothermal treatment. In the ice templating method, fairly premature hydrogels must be used to obtain microhoneycombs, so the nanoparticles which form the material tend to be small. Naturally, the nanopores, which are formed within and between such nanoparticles, tend to become small. However, it was confirmed that nanopores can be developed by treating the microhoneycombs hydrothermally, as can be seen in the results of samples MH-3 and MH-7, microhoneycombs which were treated hydrothermally for 3 days and 7days, respectively.

As in other systems, the morphology which appears during freezing can be controlled by adjusting the degree of sol-gel polymerization of the parent hydrogel. Interestingly, as shown in Fig. 12, formation of a self-standing bundle of partly connected polygonal fibers was observed during the transformation from microhoneycomb to independent polygonal fibers. It was also confirmed that the sizes of $\mu \mathrm{m}$ scale structures, such as the channel size of microhoneycombs and the diameter of microfibers, can be controlled 
by adjusting freezing conditions.

It was found that RF hydrogels obtained through ice templating can be successfully converted to carbon through drying followed by carbonization. Figure 13 shows a typical microhoneycomb, prior to and after carbonization. Although slight shrinkage occurs, it was found that the samples can maintain their unique morphologies during carbonization. Figures 14 and 15 respectively show the nitrogen adsorption-desorption isotherms of typical carbon microhoneycombs, and the mesopore size distributions derived from the isotherms. The sample prepared using a RF hydrogel without hydrothermal treatment, $\mathrm{CMH}-0$, shows a type I isotherm, indicating that this sample is microporous. However, it was found that by using RF hydrogels which were treated hydrothermally, mesoporous carbon microhoneycombs can be obtained, which can be confirmed in the isotherms and mesopore size distributions of the samples, $\mathrm{CMH}-3$ and CMH-7, typical samples prepared using hydrothermally treated precursors. Therefore it can be concluded that the ice templating method also opens a new route to synthesize porous carbons possessing both a unique morphology and a hierarchical pore system.

It is known that the unique properties of metal oxides can be improved by combining them with a different metal oxide. Such binary metal oxides can also be synthesized through sol-gel polycondensation. Therefore, finally we checked the applicability of ice templating to binary metal oxides [25].

We selected silica-alumina to represent this category. Silica-alumina typically has a high surface area, so is widely used as adsorbents and catalysts supports. They also show solid acidity, so they can be used as catalysts. Several methods to synthesize silica-alumina have been developed, but in this work we adopted a method which uses inexpensive commercial chemicals, sodium silicate solution and aluminum nitrate, as the $\mathrm{Si}$ and $\mathrm{Al}$ source, respectively. Aluminium nitrate is thought to affect the sol-gel polycondensation of silica. It has been reported that $\mathrm{Al}$ species easily form an $\mathrm{Al}-\mathrm{O}-\mathrm{Si}$ polymeric network during the sol-gel stage when the synthesis $\mathrm{pH}$ is around $2[30]$. When silica gels are synthesized using the ice templating method, the synthesis $\mathrm{pH}$ is typically close to 2 . Therefore, it is assumed that $\mathrm{Al}$ atoms can be easily incorporated into the silica framework during the sol-gel stage and form an $\mathrm{Al}-\mathrm{O}-\mathrm{Si}$ polymeric network.

First, a sodium silicate solution was prepared following the method adopted for silica gel synthesis. Next, aluminum nitrate was added to the solution, and the $\mathrm{Si} / \mathrm{Al}$ molar ratio of the solution was adjusted to a desired value. The resulting clear sol was cast in a Teflon ${ }^{\circledR}$ mold, and the mold was kept at a constant 
temperature. After the sol transformed to a gel, it was further aged for a certain period. The resulting rod-shaped hydrogels were removed from the molds and were thoroughly washed with pure water. Then the hydrogels were placed into polypropylene tubes filled with pure water, and the tubes were frozen unidirectionally, by dipping them into a cold bath at a constant rate. After freeze drying, the samples were calcined in an air flow.

It was found that macroporous monoliths of silica-alumina can be obtained when fairly soft hydrogels were used as the precursor. Figure 16 (a) shows an overall image and a SEM image of the cross-section of a typical cylindrical monolith. On the other hand, when fairly firm hydrogels were used as the precursor, polygonal fibers were obtained, as in the case of silica gel synthesis (Fig. 16 (b)).

The morphology of silica alumina monoliths was quite different from that of silica microhoneycombs. Although the cross-sections of silica alumina monoliths show voids with polygonal cross-sections, such $\mu \mathrm{m}$-sized channels were curved and interconnected. This means that their template ice crystals were also curved and interconnected. The morphology of such ice crystals resembles that of ice crystals generated through the simple freezing of hydrogels. When hydrogels are frozen by simply immersing them into a cold bath, ice crystals randomly grow within them. This is because the thermal gradient and the crystal growth rate at the freezing interface are not constant; therefore the ice crystals which grow within the hydrogel become non-uniform spheres which are randomly interconnected. On the other hand, in unidirectional freezing, the thermal gradient and the growth rate of the ice crystals at the freezing interface are almost constant. Unstable ice crystal growth at the freezing interface might have been caused by impurities [31].

Though the macropore structure of the silica alumina monoliths is not a perfect microhoneycomb, it still can be used as a macroporous monolith. It was confirmed that the macropores are highly interconnected and extend through the whole monolith, since a droplet of ink applied to one end of the monolith easily spreads to the other end. Thus, the obtained $\mathrm{SiO}_{2}-\mathrm{Al}_{2} \mathrm{O}_{3}$ cryogel monoliths have interconnected macropores and thin walls, where diffusion paths are much shorter than those in conventional particles.

The nitrogen adsorption-desorption isotherms and mesopore size distributions of typical silica-alumina monoliths are shown in Figs. 17 and 18, respectively. It can be noticed that the monoliths have developed micropores and mesopores within their structure. Therefore, although not as ordered as in the cases of 
silica and RF, the monoliths have a hierarchical pore system within them. It was found that the size and volume of mesopores tend to increase with the increase in the aging time prior to freezing, and with the decrease in the $\mathrm{Si} / \mathrm{Al}$ ratio.

Next we investigated how the aluminum atoms are distributed within the obtained materials. Figure 19 shows a micrograph of a typical silica-alumina monolith, and the corresponding EDS mappings of $\mathrm{Si}$ and $\mathrm{Al}$ atoms. It can be confirmed that $\mathrm{Al}$ atoms are homogeneously dispersed without local aggregation in the silica framework. This was also confirmed through Raman spectroscopy, and ${ }^{27} \mathrm{Al}$ MAS-NMR.

It was confirmed that in the silica-alumina samples, Al atoms are incorporated into the silica framework by forming an Al-O-Si polymeric network. Therefore these samples are expected to show Brønsted acidity. We characterized the acidity of typical samples using the $a c$-TPD method [32]. Figure 20 shows $\mathrm{NH}_{3}$-TPD spectra of typical monolith samples. Note that the spectra only show desorption from acid sites. It is clearly seen that each sample has acid sites, which indirectly proves that $\mathrm{Al}-\mathrm{O}-\mathrm{Si}$ bonds exist within the samples. Moreover, it was found that the sample synthesized under a lower $\mathrm{Si} / \mathrm{Al}$ ratio, SAMH-4, has a larger amount of acid sites than the other samples. This result indicates that it is possible to control the amount of acid sites by controlling the $\mathrm{Si} / \mathrm{Al}$ ratio in the parent sols.

Through the results shown above, it can be concluded that the ice templating method can be applied to a wide variety of hydrogels, and can provide inorganic as well as organic materials with unique hierarchical pore systems.

\section{CONCLUSION}

This article overviews the outline of the ice templating method, a newly developed method to obtain porous materials with a unique hierarchical pore system of micro-, meso- and macropores. In this method, the morphology, $\mu \mathrm{m}$ scale structures and $\mathrm{nm}$ scale structures of the materials obtained through ice templating can be independently controlled by adjusting synthesis conditions. Therefore, the pore system in materials obtained through ice templating can be tuned so that they can show their maximum performance in the processes they are going to be used in. General features of materials obtained through this method are:

(1) All of the materials obtained through this method have straight $\mu \mathrm{m}$-sized macropores, if the space 
formed between aligned fibers and sheets are also considered as macropores.

(2) The lengths of the nanopores are limited by the thicknesses of the sheets and walls, or the diameters of the materials, therefore are extremely short.

Therefore, if the materials obtained through this method are to be used to treat fluids, the pressure drop which is likely to occur can be suppressed to extremely low levels, even though the diffusion paths in the material are extremely short. This feature cannot be achieved using porous materials of conventional morphologies. As this method can be applied to a wide variety of hydrogels, inorganic as well as organic, it is widely expected to provide unique porous materials with enhanced properties which can be used as adsorbents and/or catalysts. 


\section{LITERATURE CITED}

[1] A. R. Studart, U. T. Gonzenbach, E. Tervoot and L. J. Gauckler, J. Am. Ceram. Soc. 89 (2006) 1771.

[2] K. Nakanishi, J. Porous Mater. 4 (1997) 67

[3] K. Nakanishi and N. Soga, J. Am. Ceram. Soc. 74 (1991) 2518.

[4] H. Minakuchi, K. Nakanishi, N. Soga, N. Ishizuka and N. Tanaka, Anal. Chem. 68 (1996) 3498.

[5] J. H. Smatt, S. Schunk and M. Linden, Chem. Mater. 15 (2003) 2354.

[6] N. Nakamura, R. Takahashi, S. Sato, T. Sodesawa and S. Yoshida, Phys. Chem. Chem. Phys. 2 (2000) 4983.

[7] O. D. Velev, T. A. Jede, R. F. Lobo and A. M. Lenhoff, Nature 389 (1997) 447.

[8] B. T. Holland, C. F. Blanford and A. Stein, Science 281 (1998) 538.

[9] O. D. Velev and E. W. Kaler, Adv. Mater. 12 (2000) 531.

[10] K. M. Kulinowski, P. Jiang, H. Vaswani and V. L. Colvin, Adv. Mater. 12 (2000) 833.

[11] A. Imhof and D. J. Pine, Nature 389 (1997) 948.

[12] V. N. Manoharan, A. Imhof, J. D. Thorne and D. J. Pine, Adv. Mater. 13 (2001) 447.

[13] J. Laurie, C. M. Bagnall, B. Harris, R. W. Jones, R. G. Cooke, R. S. Russell-Floyd, T. H. Wang and F. W. Hammett, J. Non-Cryst. Solids 147-148 (1992) 320.

[14] M. J. Statham, F. Hammett, B. Harris, R. G. Cooke, R. M. Jordan and A. Roche, J. Sol-Gel Sci. Technol. 13 (1998) 171.

[15] D. Koch, L. Andresen, T. Schmedders and G. Grathwohl, J. Sol-Gel Sci. Technol. 26 (2003) 149.

[16] W. Mahler and M. F. Bechtold, Nature 285 (1980) 27.

[17] T. Kokubo, Y. Teranishi and T. Maki, J. Non-Cryst. Solids 56 (1983) 411.

[18] T. Maki and S. Sakka, J. Mat. Sci. Lett. 5 (1986) 28.

[19] S. R. Mukai, H. Nishihara and H. Tamon, Microporous Mesoporous Mater., 63 (2003) 43.

[20] S. R. Mukai, H. Nishinara and H. Tamon, Chem. Commun. (2004) 874.

[21] H. Nishihara, S. R. Mukai and H. Tamon, Carbon 42 (2004) 899.

[22] S. R. Mukai, H. Nishihara, S. Shichi and H. Tamon, Chem. Mater. 16 (2004) 4987.

[23] H. Nishinara, S. R. Mukai, D. Yamashita and H. Tamon, Chem. Mater. 17 (2005) 683.

[24] S. R. Mukai, H. Nishihara, T. Yoshida, K. Taniguchi and H. Tamon, Carbon 43 (2005) 1563. 
[25] H. Nishihara, S. R. Mukai, Y. Fujii, T. Tago, T. Masuda and H. Tamon, J. Mater. Chem. 16 (2006) 3231.

[26] M. C. Flemings, Solidification Processing, McGraw-Hill, New York (1974).

[27] K. Nakanishi, R. Takahashi, T. Nagakane, K. Kitayama, N. Koheiya, H. Shikata and N. Soga, J. Sol-Gel Sci. Technol. 17 (2000) 191.

[28] R. W. Pekala, J. Mater. Sci. 24 (1989) 3221.

[29] R. W. Pekala, C. T. Alviso, R. F. Kong and S. S. Hulsey, J. Non-Cryst. Solids, 145 (1992) 90.

[30] C. Hernandez and A. C. Pierre, Langmuir 16 (2000) 530.

[31] B. Chalmers, Principles of Solidification, Wiley, New York (1964).

[32] T. Masuda, Y. Fujikata, S. R. Mukai and K. Hashimoto, Appl. Catal. A, 165 (1997) 57. 


\section{FIGURE CAPTIONS}

Fig. 1 Morphologies of typical silica samples obtained through ice templating ((a) lamella sheets, (b) flat fibers, (c) monolithic microhoneycomb, (d) polygonal fibers)

Fig. 2 Confocal laser micrograph of silica polygonal fibers obtained through ice templating

Fig. 3 Nanostructure of silica polygonal fibers

((a) SEM image, (b) $\mathrm{N}_{2}$ adsorption-desorption isotherms (77 K) and mesopore size distribution derived from the isotherms (inset), $S_{\mathrm{BET}}$ : BET surface area)

Fig. 4 Morphology and structure of a typical silica microhoneycomb

((a) an overall image, SEM images of (b) cross-section, (c) channel structure, (d) vertical section)

Fig. 5 Nanostructure of honeycomb wall

((a) SEM image, (b) $\mathrm{N}_{2}$ adsorption-desorption isotherms $(77 \mathrm{~K}$ ) and mesopore size distribution derived from the isotherms (inset), $S_{\mathrm{BET}}$ : BET surface area)

Fig. 6 Relationship between the average channel size of silica microhoneycombs and freezing parameter $\left\{v_{\mathrm{f}}\left(T_{\mathrm{r}}-T_{\mathrm{f}}\right)\right\}^{-1}$

( $v_{\mathrm{f}}:$ immersion rate, $T_{\mathrm{r}}$ : room temperature, $T_{\mathrm{f}}$ : freezing temperature)

Fig. $7 \quad \mathrm{~N}_{2}$ adsorption-desorption isotherms $(77 \mathrm{~K})$ of silica microhoneycombs hydrothermally treated for 5 days in $\mathrm{NH}_{3} a q$. of different concentrations $C_{\mathrm{N}}$ and different temperatures $T_{\mathrm{ht}}$

((a) No treatment, (b) $C_{\mathrm{N}}=0.1 \mathrm{~mol} / \mathrm{L}, T_{\mathrm{ht}}=298 \mathrm{~K}$, (c) $C_{\mathrm{N}}=0.1 \mathrm{~mol} / \mathrm{L}$ and $T_{\mathrm{ht}}=323 \mathrm{~K}$, (d) $C_{\mathrm{N}}=$ $1.0 \mathrm{~mol} / \mathrm{L}$ and $T_{\mathrm{ht}}=323 \mathrm{~K}$, (e) $C_{\mathrm{N}}=1.0 \mathrm{~mol} / \mathrm{L}$ and $T_{\mathrm{ht}}=363 \mathrm{~K}, S_{\mathrm{BET}}$ : BET surface area)

Fig. 8 Mesopore size distributions of silica microhoneycombs hydrothermally treated for 5 days in $\mathrm{NH}_{3}$ $a q$. of different concentrations $C_{\mathrm{N}}$ and different temperatures $T_{\mathrm{ht}}$

((a) No treatment, (b) $C_{\mathrm{N}}=0.1 \mathrm{~mol} / \mathrm{L}, T_{\mathrm{ht}}=298 \mathrm{~K}$, (c) $C_{\mathrm{N}}=0.1 \mathrm{~mol} / \mathrm{L}$ and $T_{\mathrm{ht}}=323 \mathrm{~K}$, (d) $C_{\mathrm{N}}=$ $1.0 \mathrm{~mol} / \mathrm{L}$ and $T_{\mathrm{ht}}=323 \mathrm{~K}$, (e) $C_{\mathrm{N}}=1.0 \mathrm{~mol} / \mathrm{L}$ and $T_{\mathrm{ht}}=363 \mathrm{~K}$ )

Fig. 9 Typical RF gels obtained through ice templating

((a), (b) microhoneycombs, (c), (d) polygonal fibers)

Fig. $10 \mathrm{~N}_{2}$ adsorption-desorption isotherms $(77 \mathrm{~K})$ of typical microhoneycomb-type RF gels $\left(S_{\mathrm{BET}}\right.$ : BET surface area)

Fig. 11 Mesopore size distributions of typical microhoneycomb-type RF gels 
Fig. 12 SEM images of a typical self-standing bundle of an RF gel

((a) cross-section, (b) enlarged image)

Fig. 13 SEM images of the cross-section of a typical carbon microhoneycomb

((a) before carbonization, (b) after carbonization)

Fig. $14 \mathrm{~N}_{2}$ adsorption-desorption isotherms $(77 \mathrm{~K})$ of typical carbon microhoneycombs $\left(S_{\mathrm{BET}}\right.$ : BET surface area)

Fig. 15 Mesopore size distributions of typical carbon microhoneycombs

Fig. 16 (a) SEM image of a typical silica-alumina monolith and (b) Confocal laser micrograph of typical silica-alumina fibers, obtained through ice templating

Fig. $17 \mathrm{~N}_{2}$ adsorption-desorption isotherms of typical silica-alumina monoliths prepared under different $\mathrm{Si} / \mathrm{Al}$ values and aging times $t_{\text {hyd }}$

$\left(\mathrm{SAMH}-1: \mathrm{Si} / \mathrm{Al}=3.8, t_{\text {hyd }}=3.8 \mathrm{~h}, \mathrm{SAMH}-3: \mathrm{Si} / \mathrm{Al}=3.8, t_{\text {hyd }}=6.6 \mathrm{~h}, \mathrm{SAMH}-4: \mathrm{Si} / \mathrm{Al}=1.9, t_{\text {hyd }}\right.$ $=4.3 \mathrm{~h}, \mathrm{SAMH}-5: \mathrm{Si} / \mathrm{Al}=1.9, t_{\mathrm{hyd}}=6.6 \mathrm{~h}, S_{\mathrm{BET}}:$ BET surface area)

Fig. 18 Mesopore size distributions of typical silica-alumina monoliths prepared under different $\mathrm{Si} / \mathrm{Al}$ values and aging times $t_{\text {hyd }}$

$\left(\mathrm{SAMH}-1: \mathrm{Si} / \mathrm{Al}=3.8, t_{\text {hyd }}=3.8 \mathrm{~h}, \mathrm{SAMH}-3: \mathrm{Si} / \mathrm{Al}=3.8, t_{\text {hyd }}=6.6 \mathrm{~h}, \mathrm{SAMH}-4: \mathrm{Si} / \mathrm{Al}=1.9, t_{\text {hyd }}\right.$ $\left.=4.3 \mathrm{~h}, \mathrm{SAMH}-5: \mathrm{Si} / \mathrm{Al}=1.9, \mathrm{t}_{\mathrm{hyd}}=6.6 \mathrm{~h}\right)$

Fig. 19 (a) An enlarged SEM image of the cross-section of a typical silica-alumina monolith and corresponding EDS mappings for (b) Si and (c) Al

Fig. $20 \mathrm{NH}_{3}$-TPD spectra of the $\mathrm{SiO}_{2}-\mathrm{Al}_{2} \mathrm{O}_{3}$ cryogel monoliths obtained by the $a c$-TPD method. 


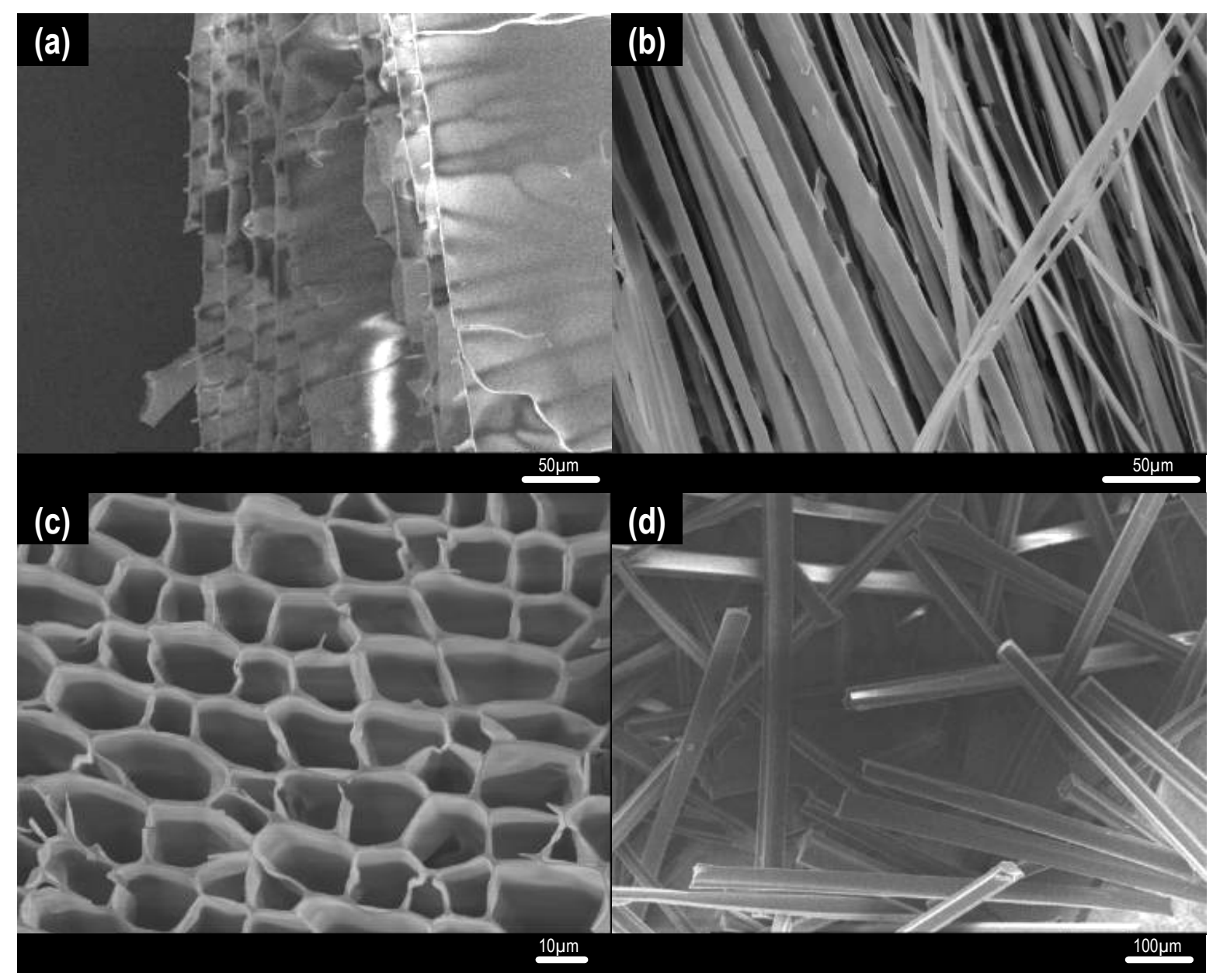

Mukai et al. Fig. 1 


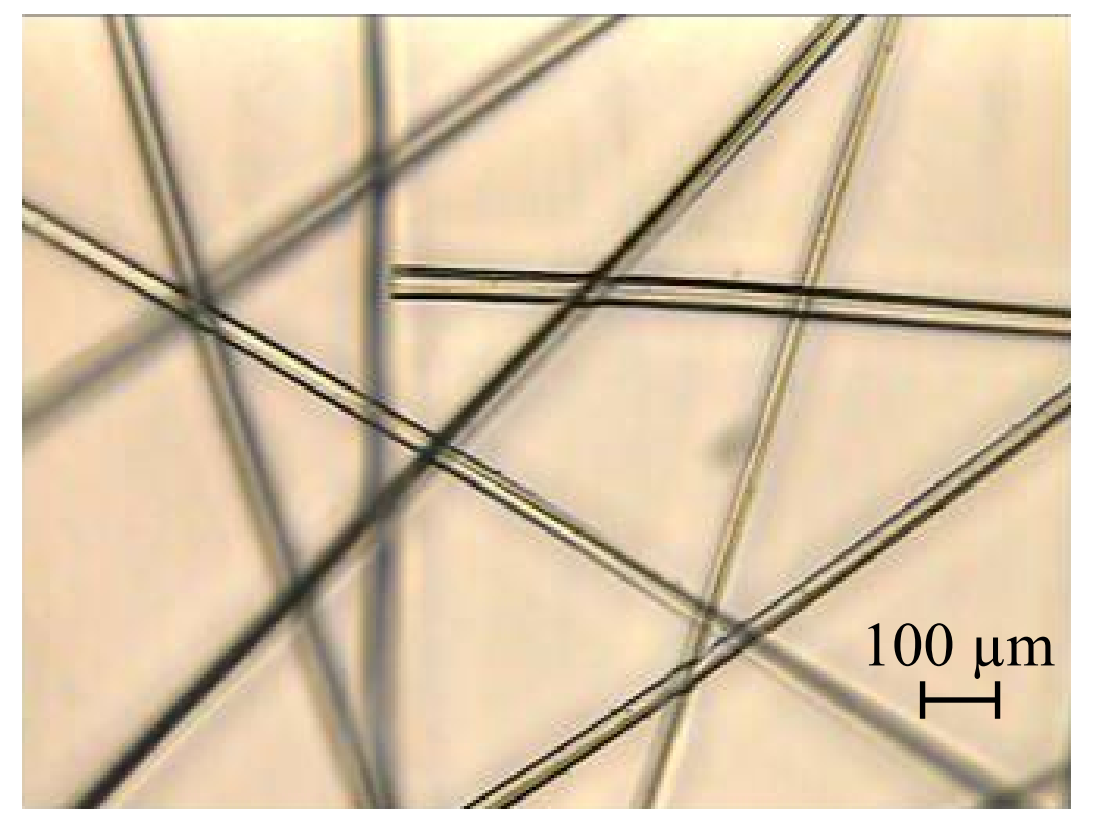

Mukai et al. Fig. 2 

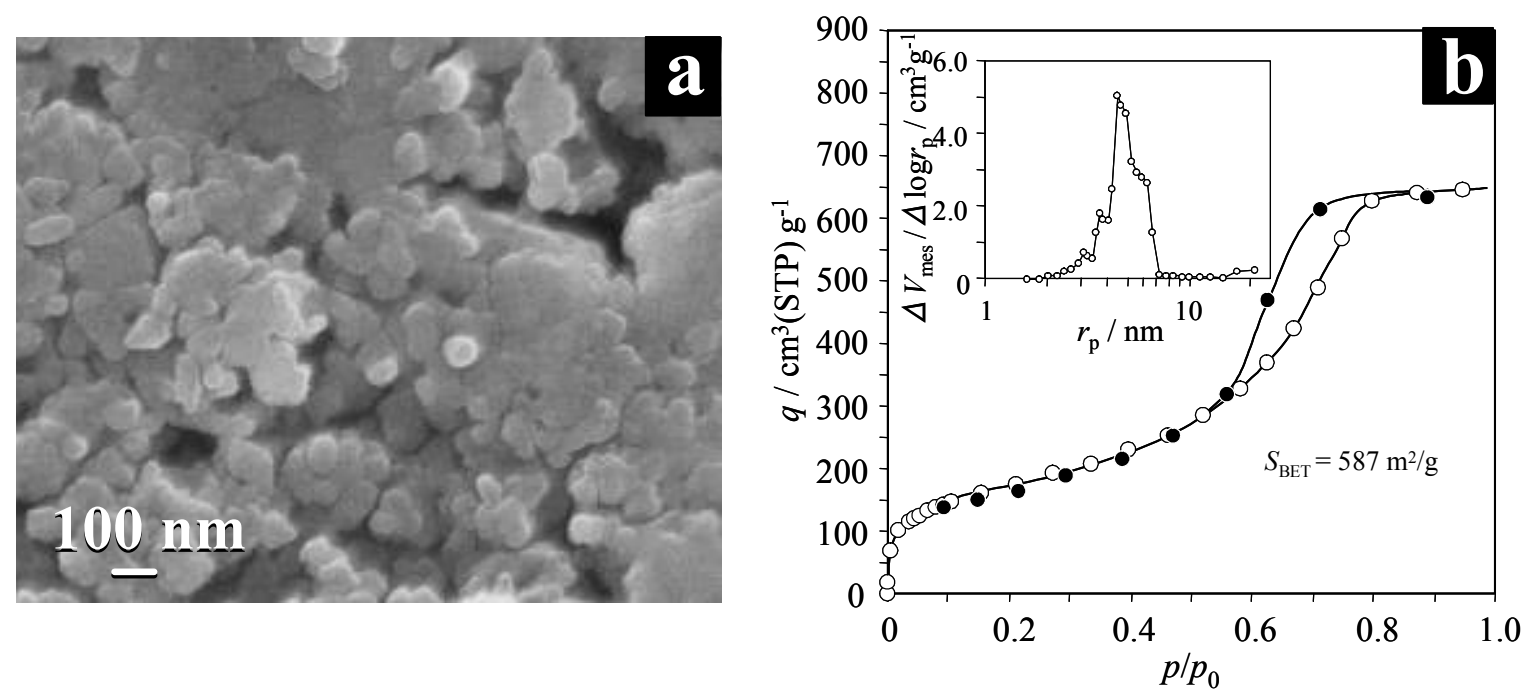

Mukai et al. Fig. 3 

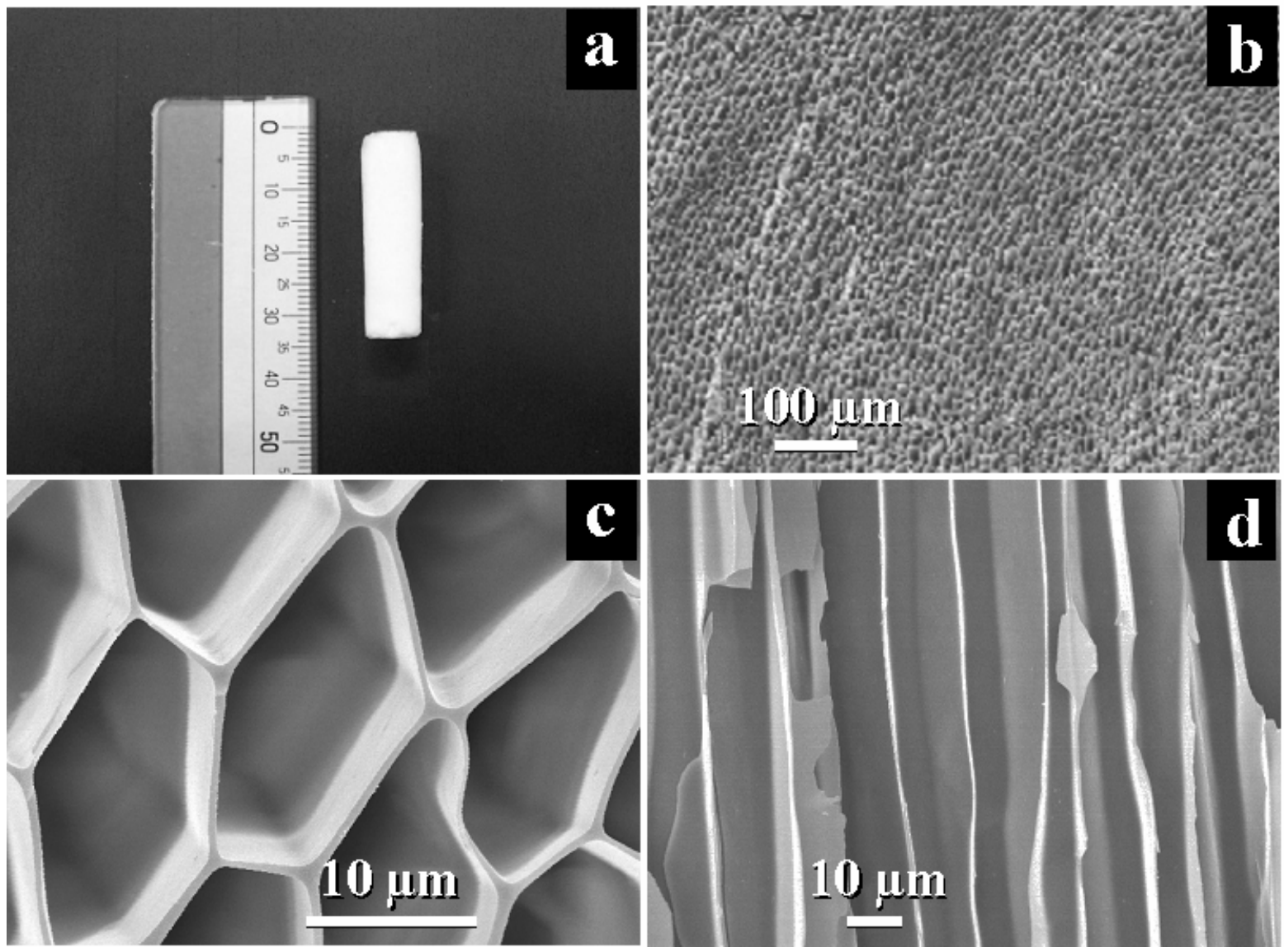

Mukai et al. Fig. 4 

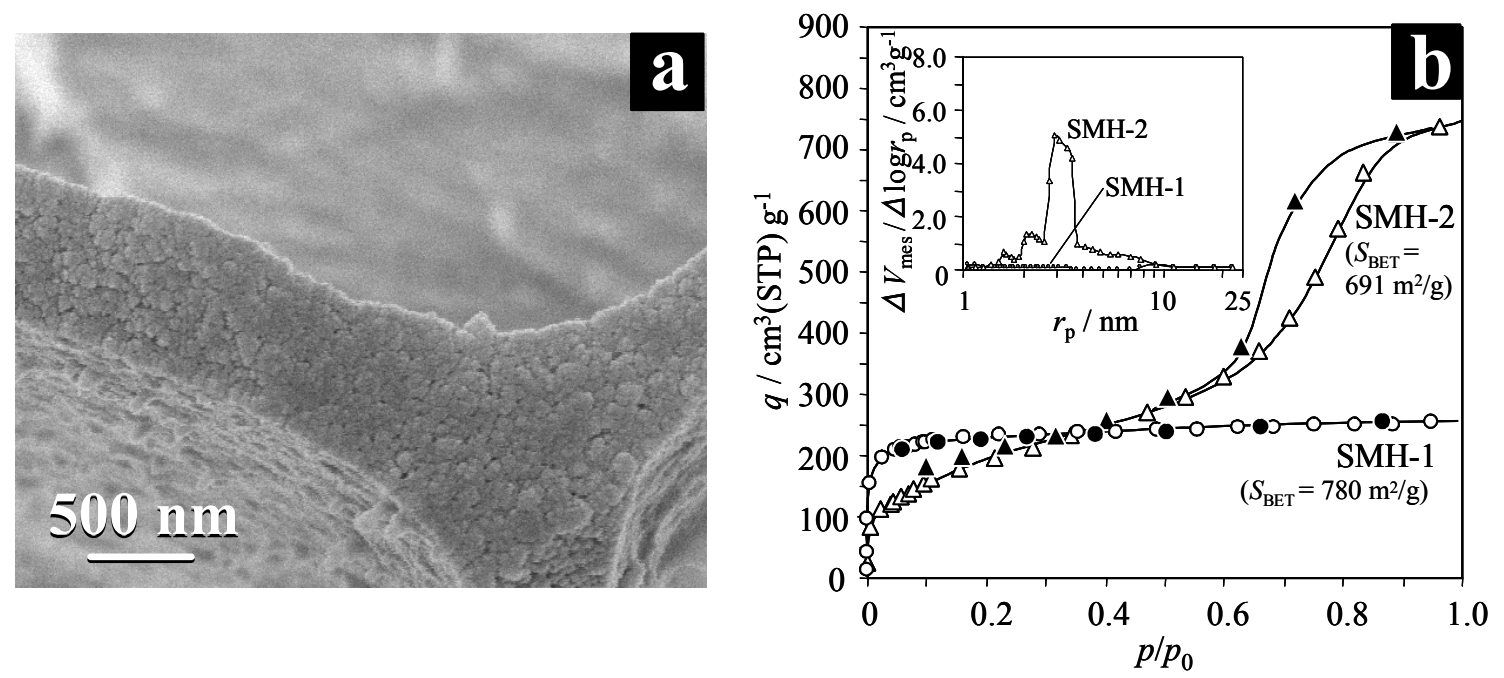

Mukai et al. Fig. 5 


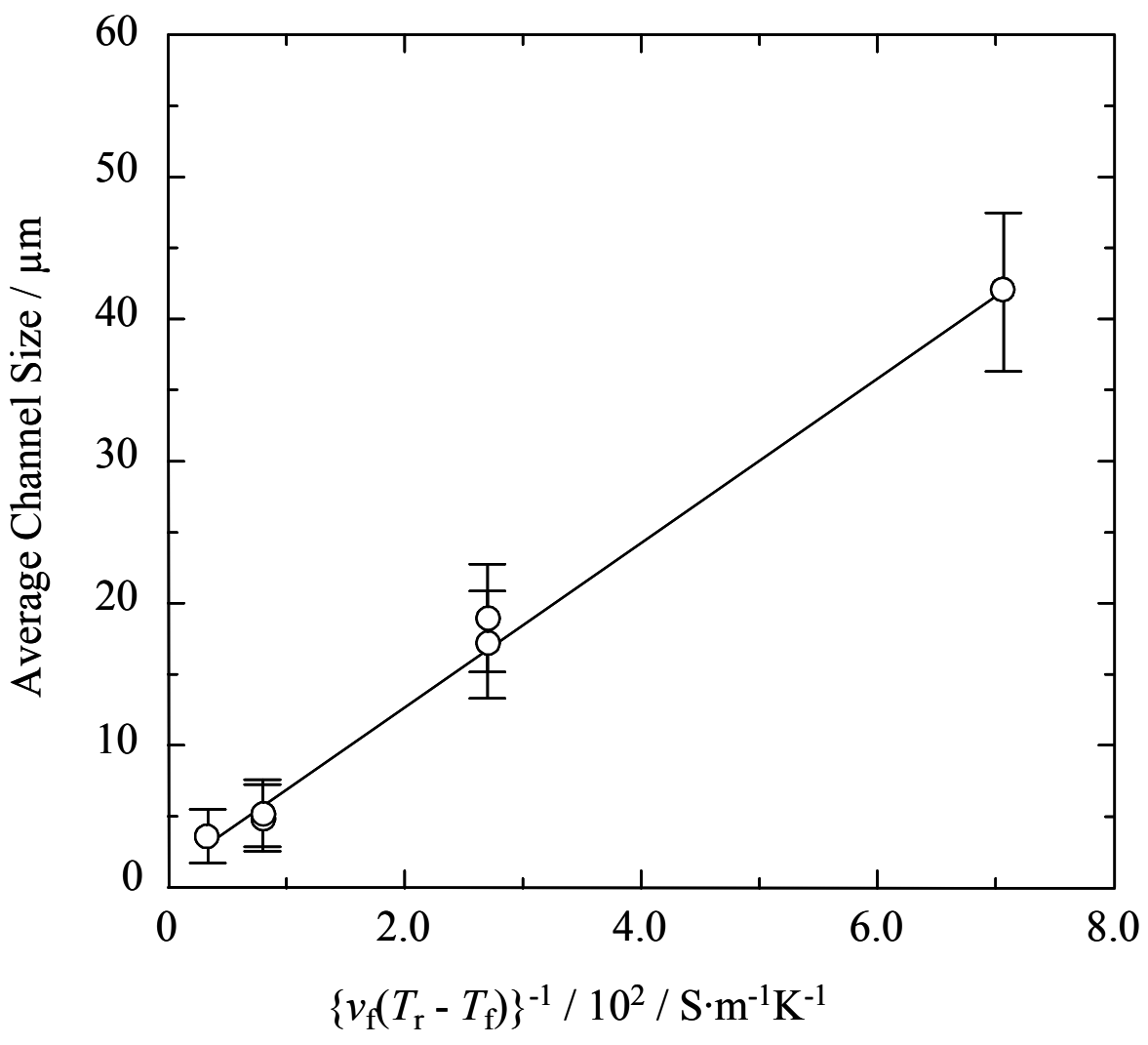

Mukai et al. Fig. 6 


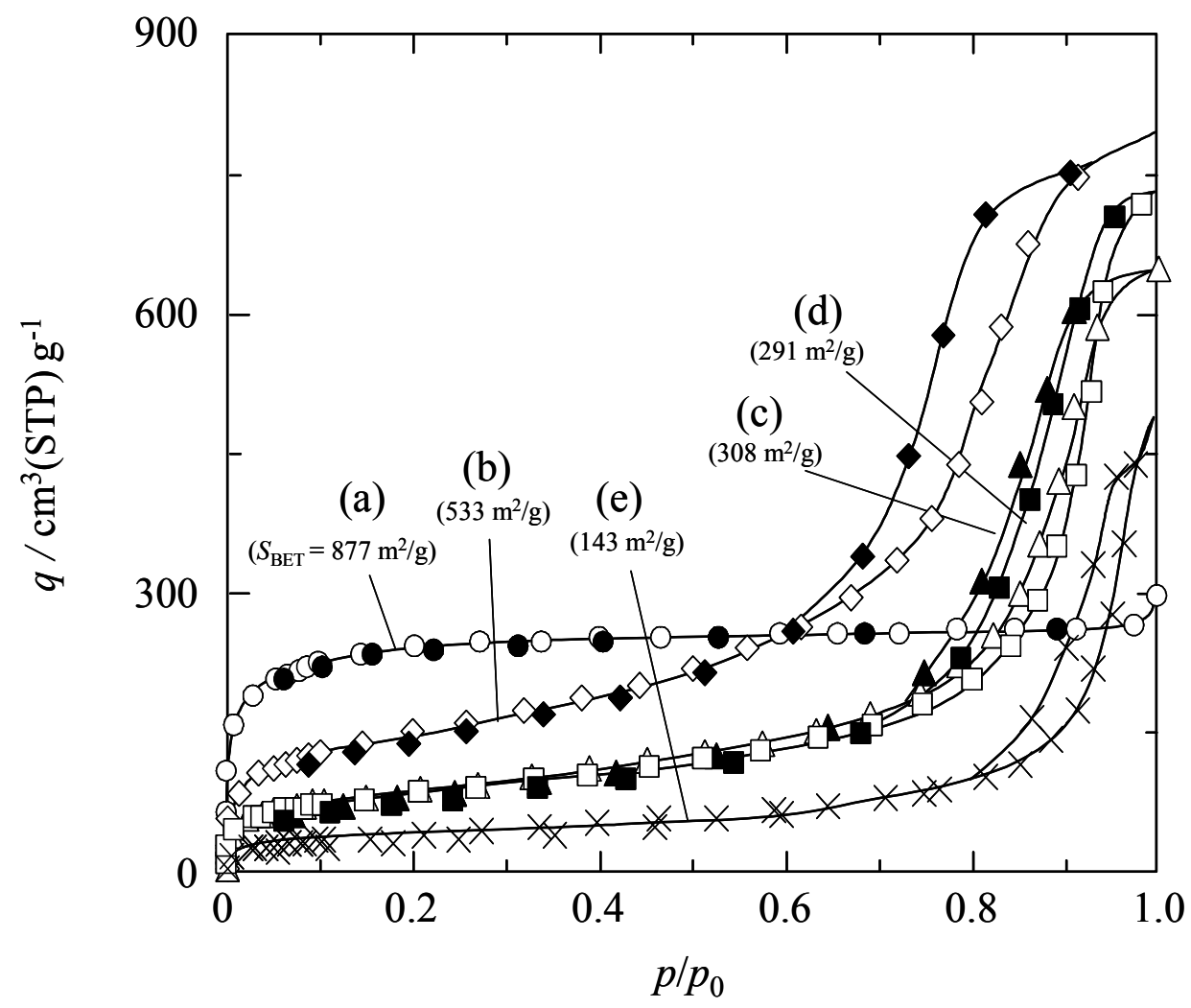

Mukai et al. Fig. 7 


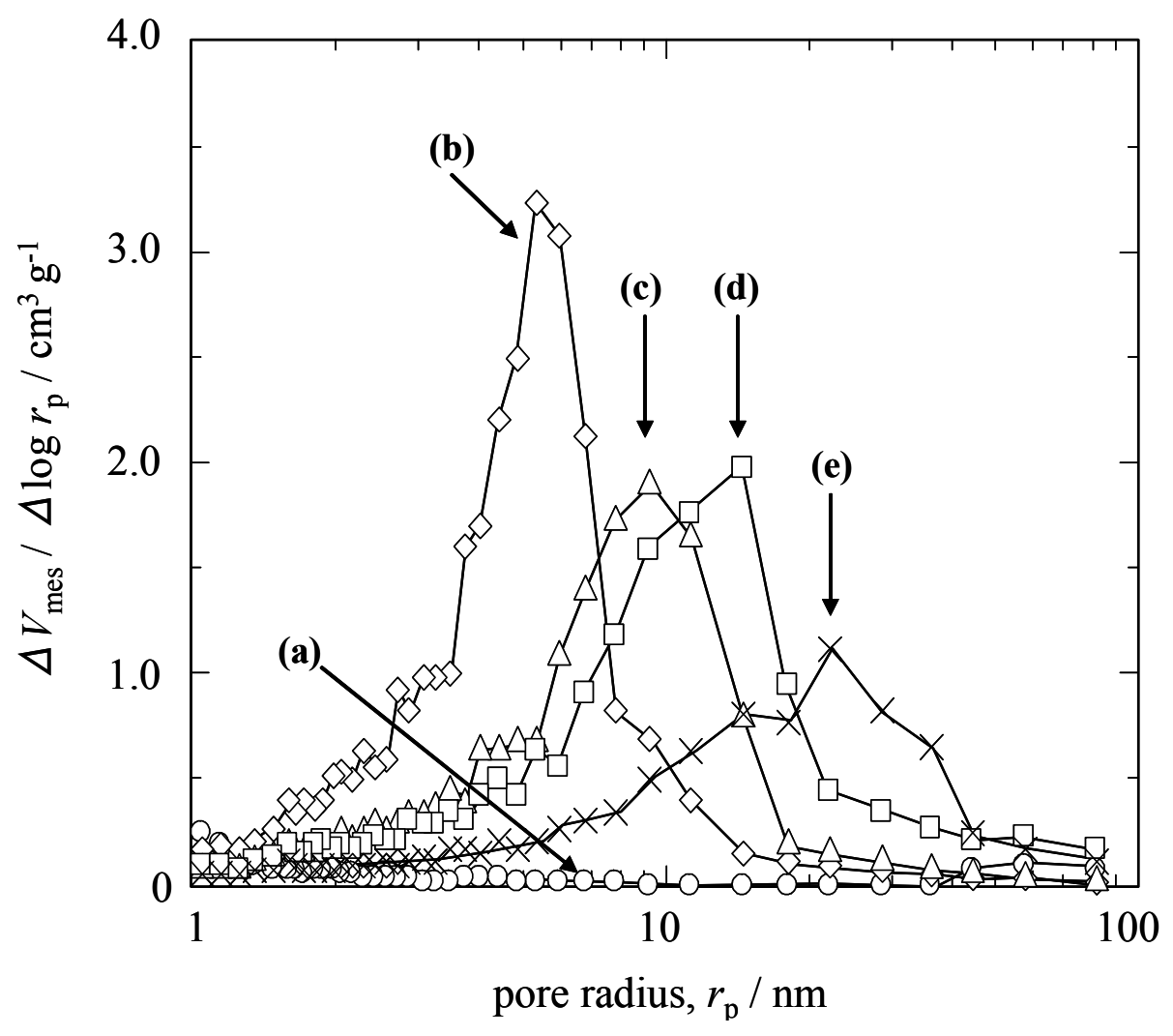

Mukai et al. Fig. 8 

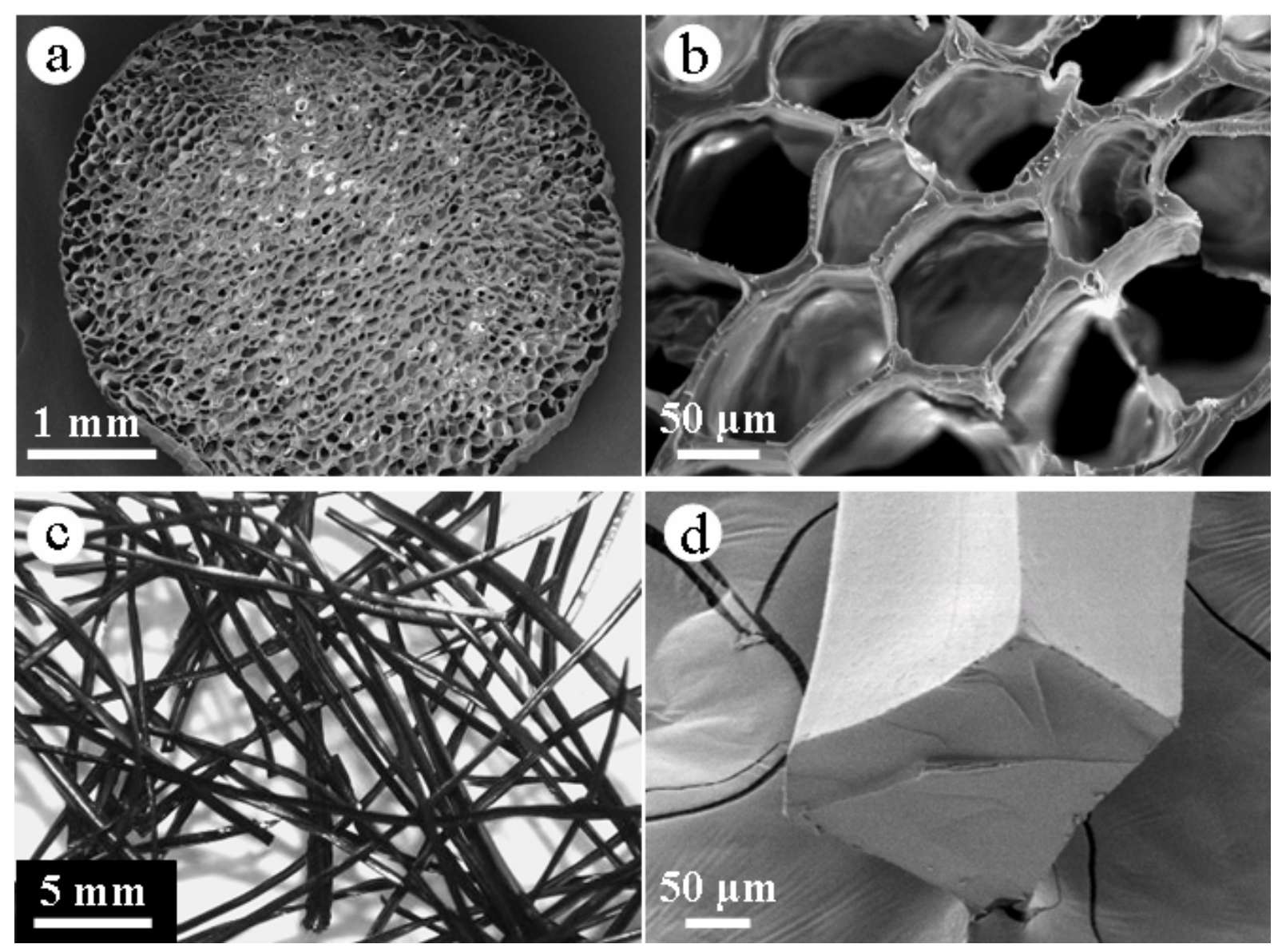

Mukai et al. Fig. 9 


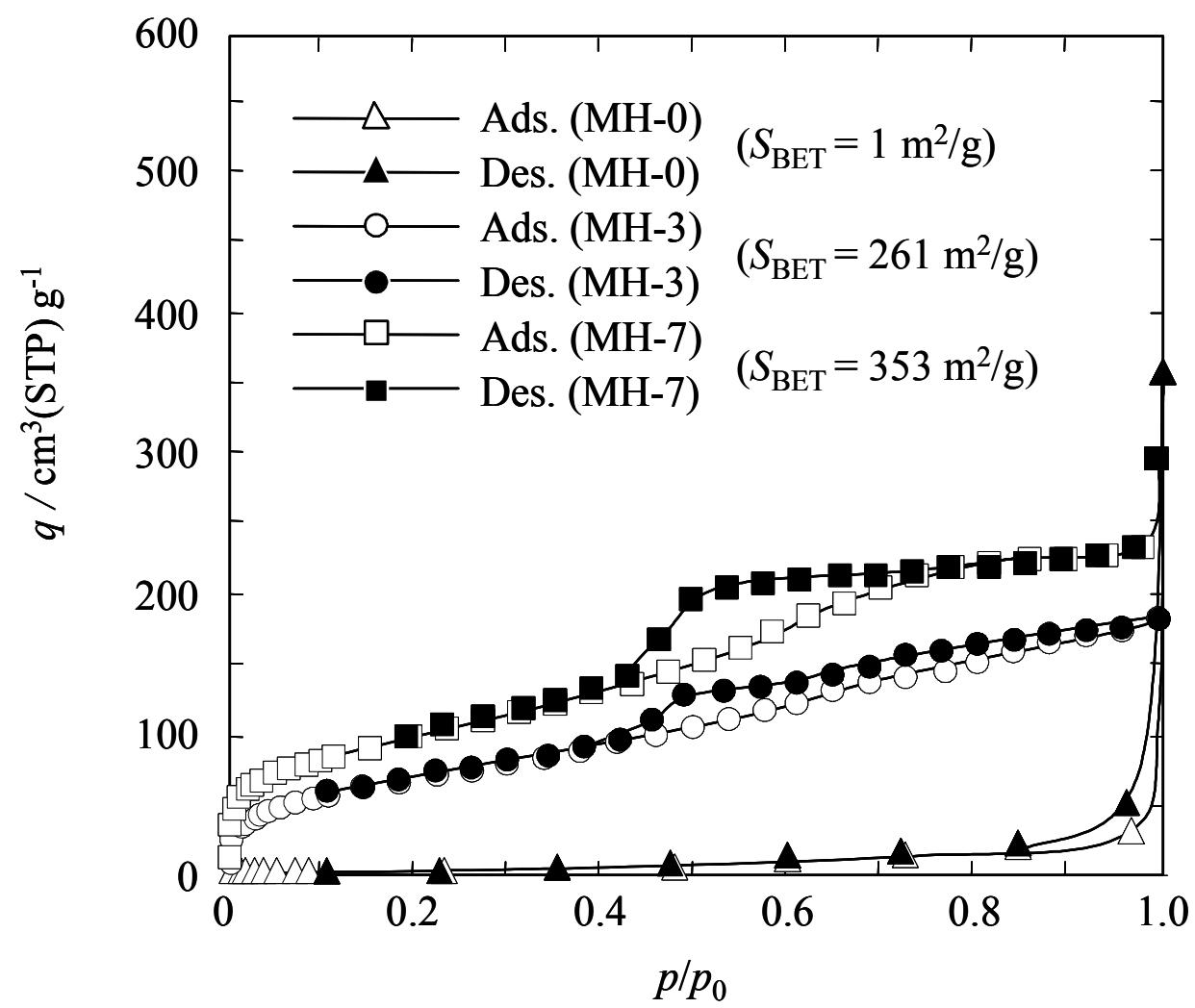

Mukai et al. Fig. 10 


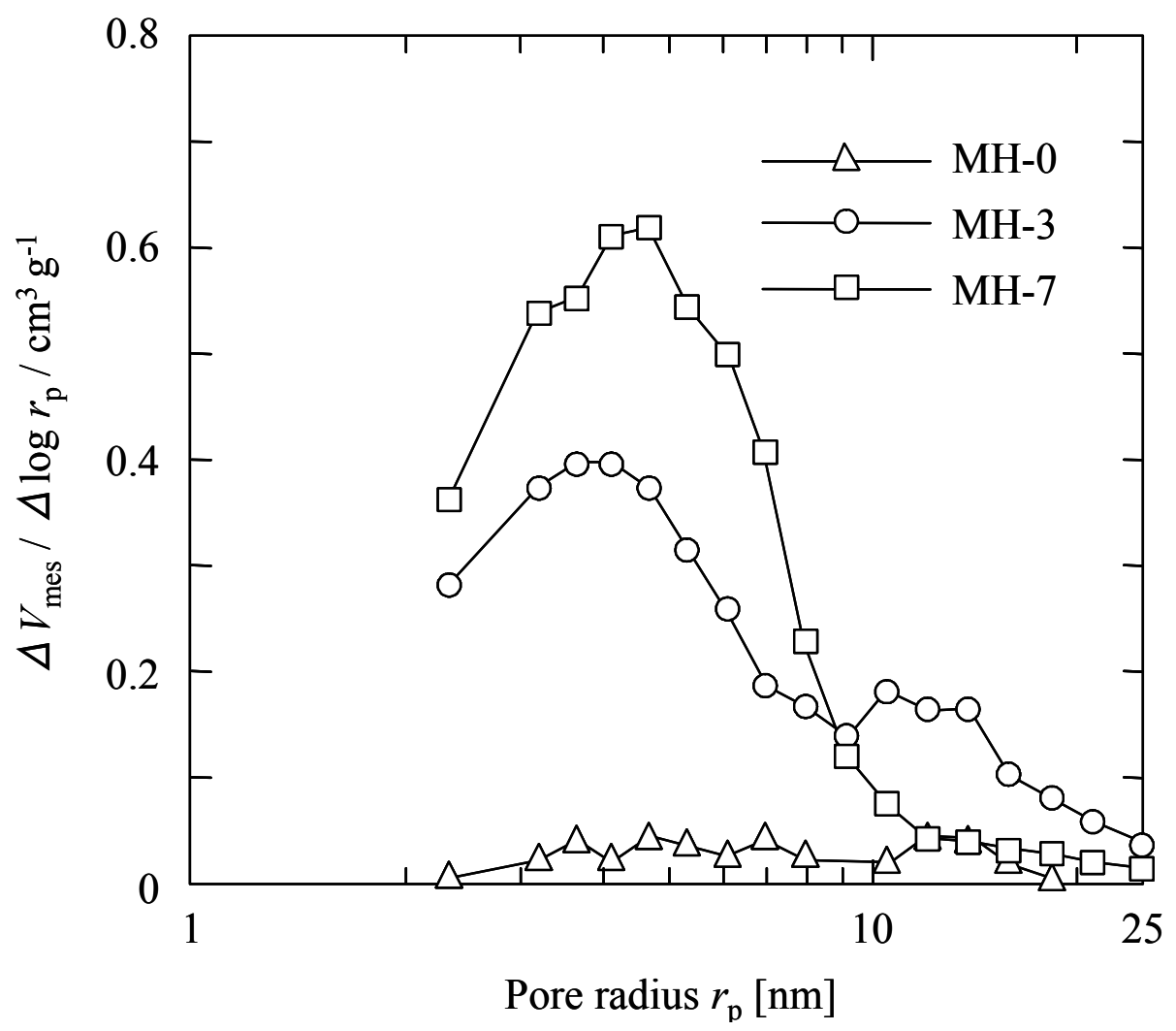

Mukai et al. Fig. 11 


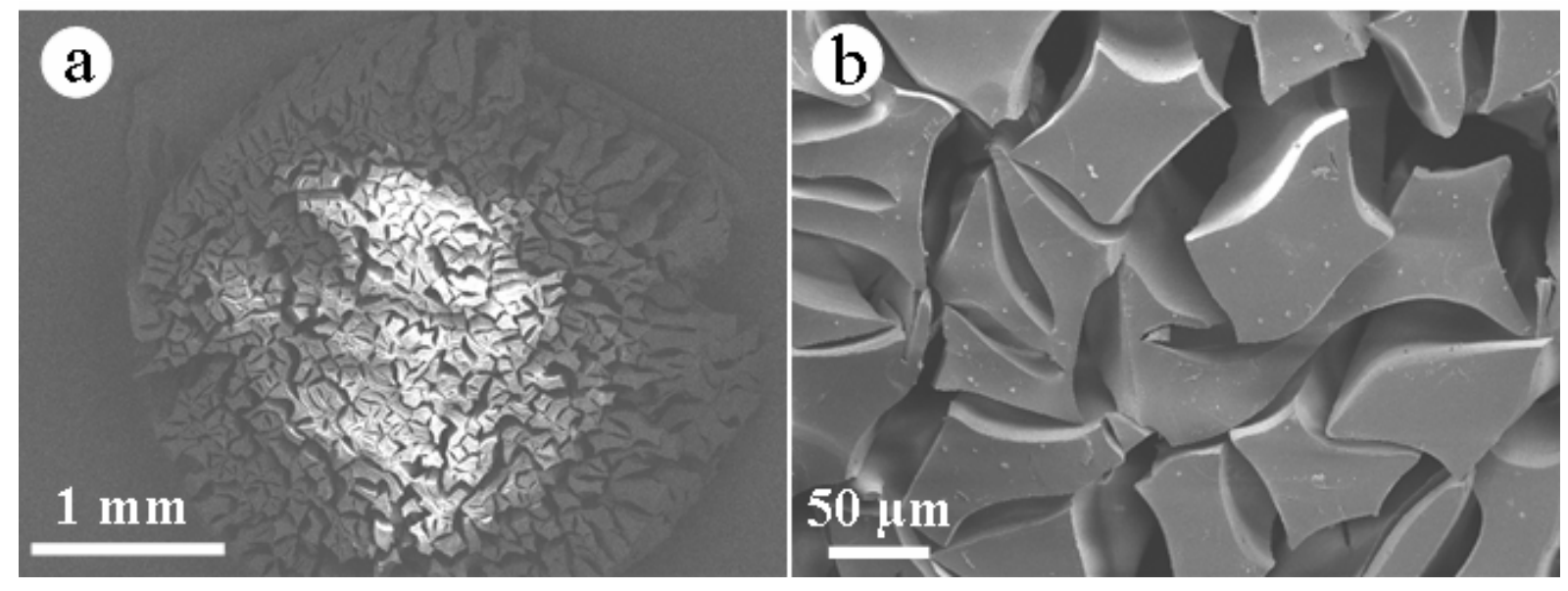

Mukai et al. Fig. 12 

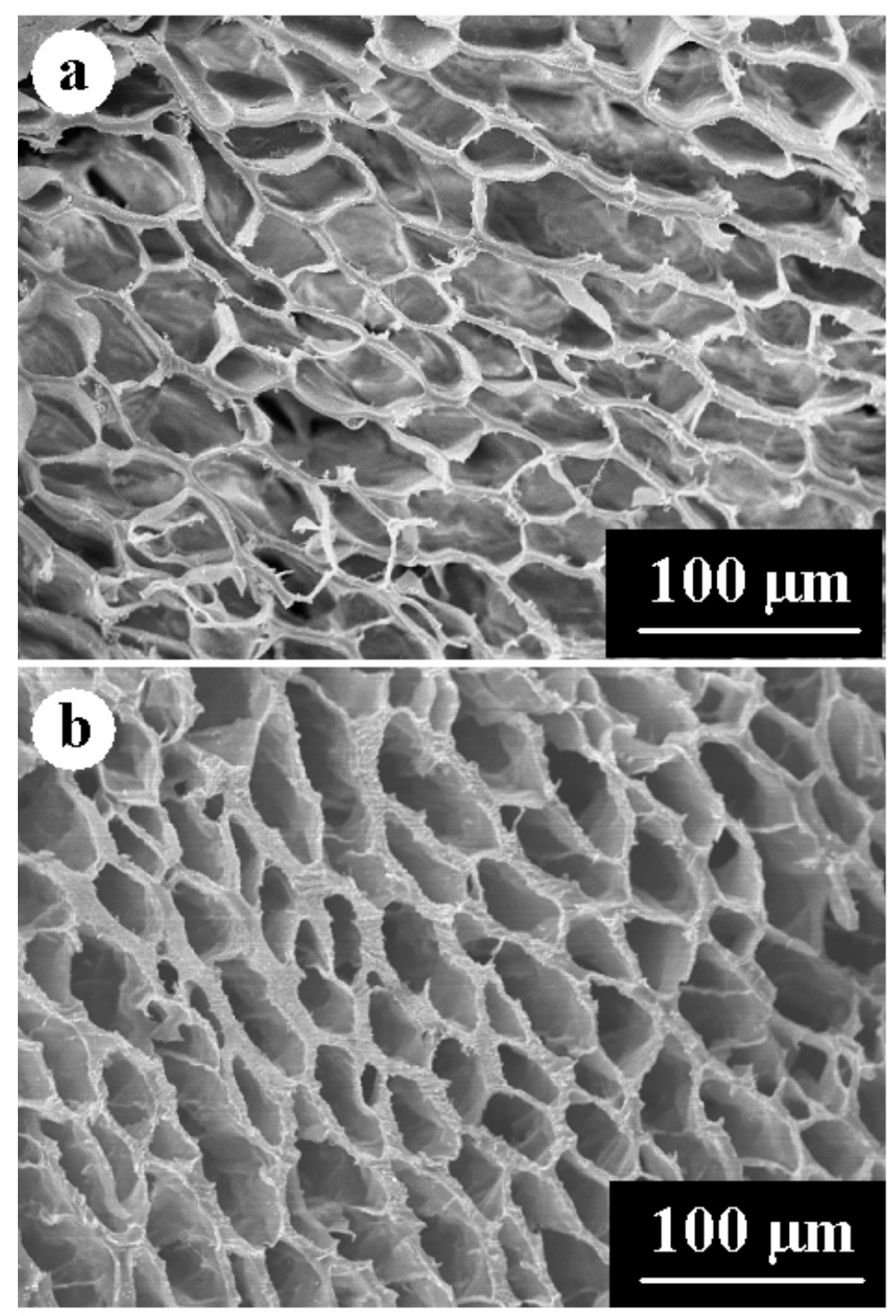

Mukai et al. Fig. 13 


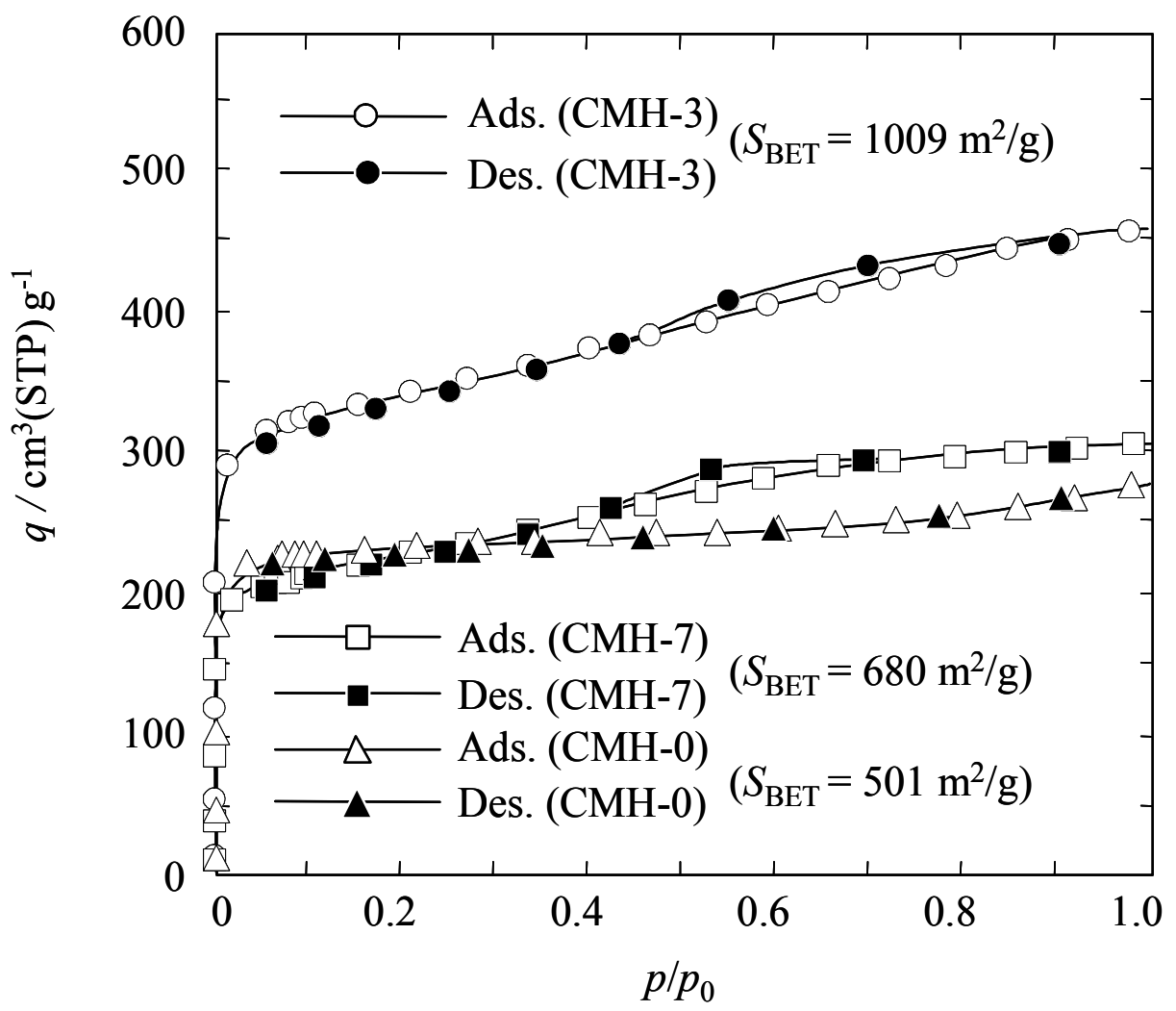

Mukai et al. Fig. 14 


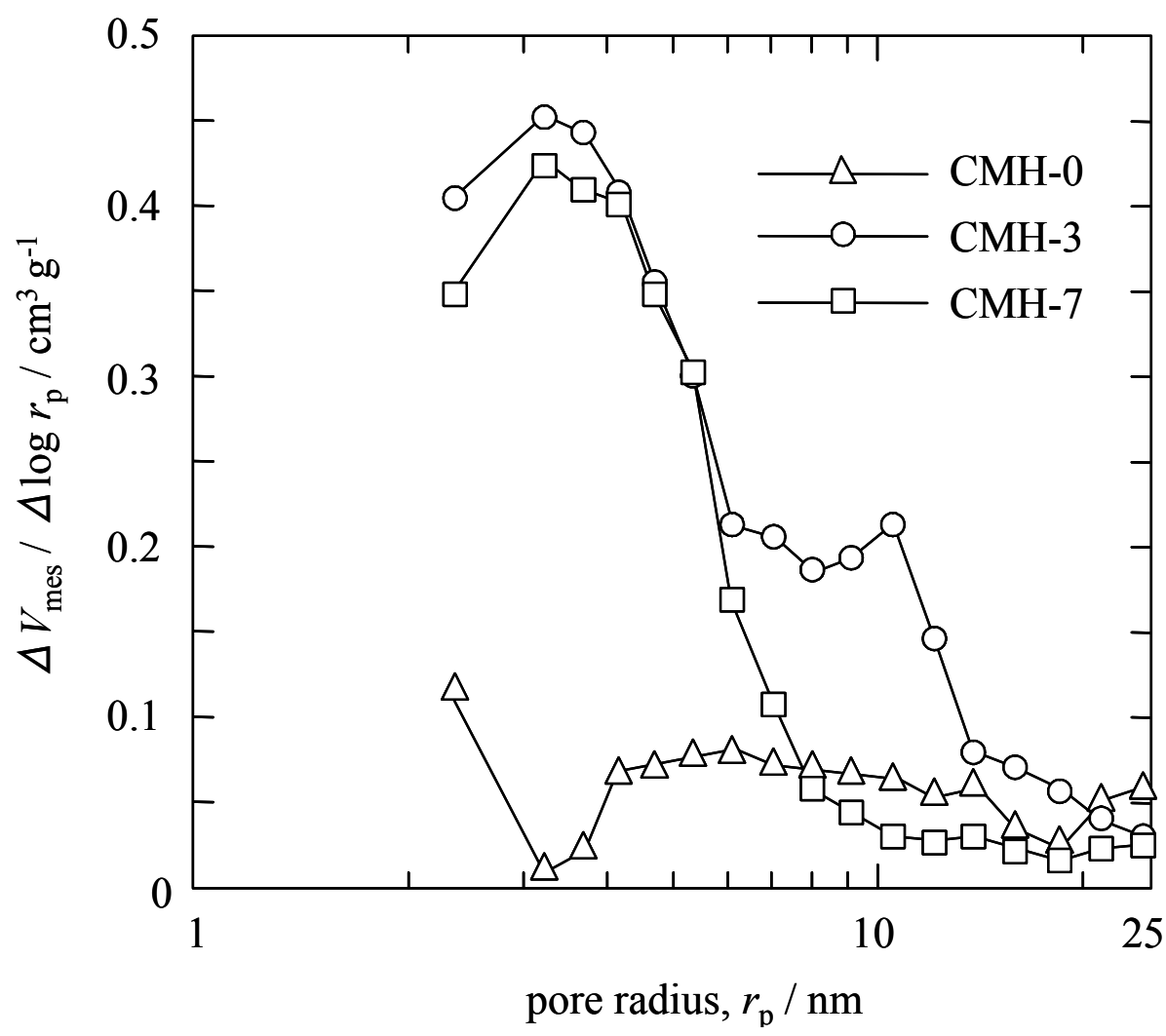

Mukai et al. Fig. 15 


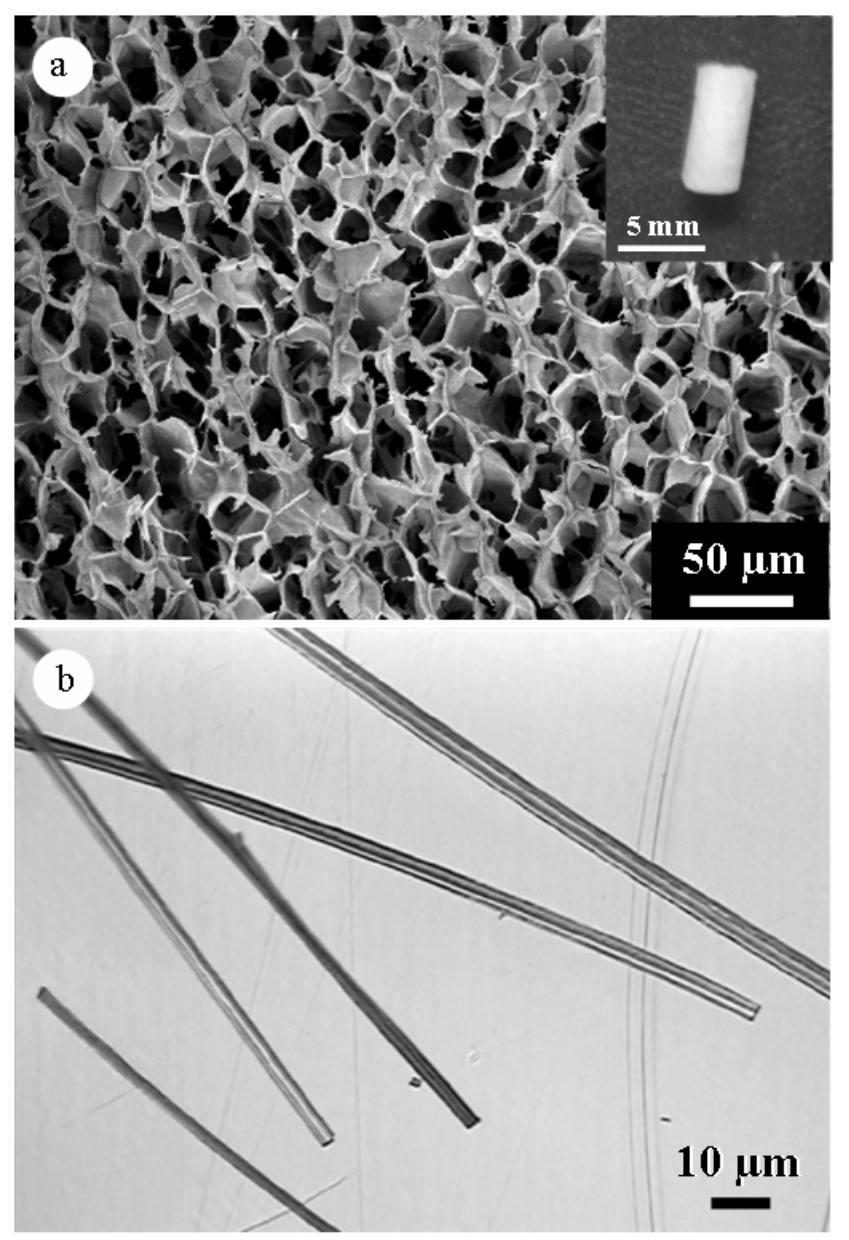

Mukai et al. Fig. 16 


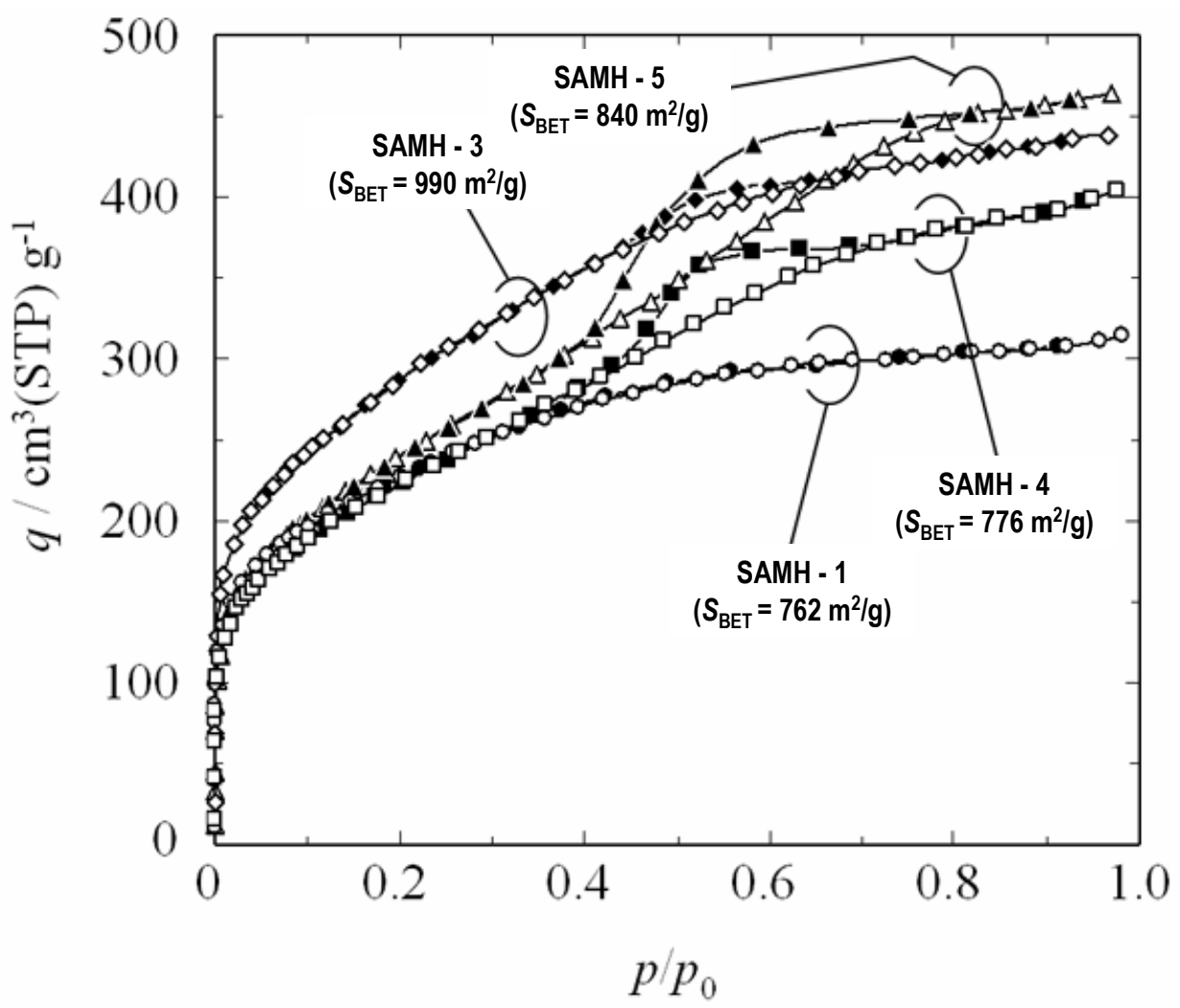

Mukai et al. Fig. 17 


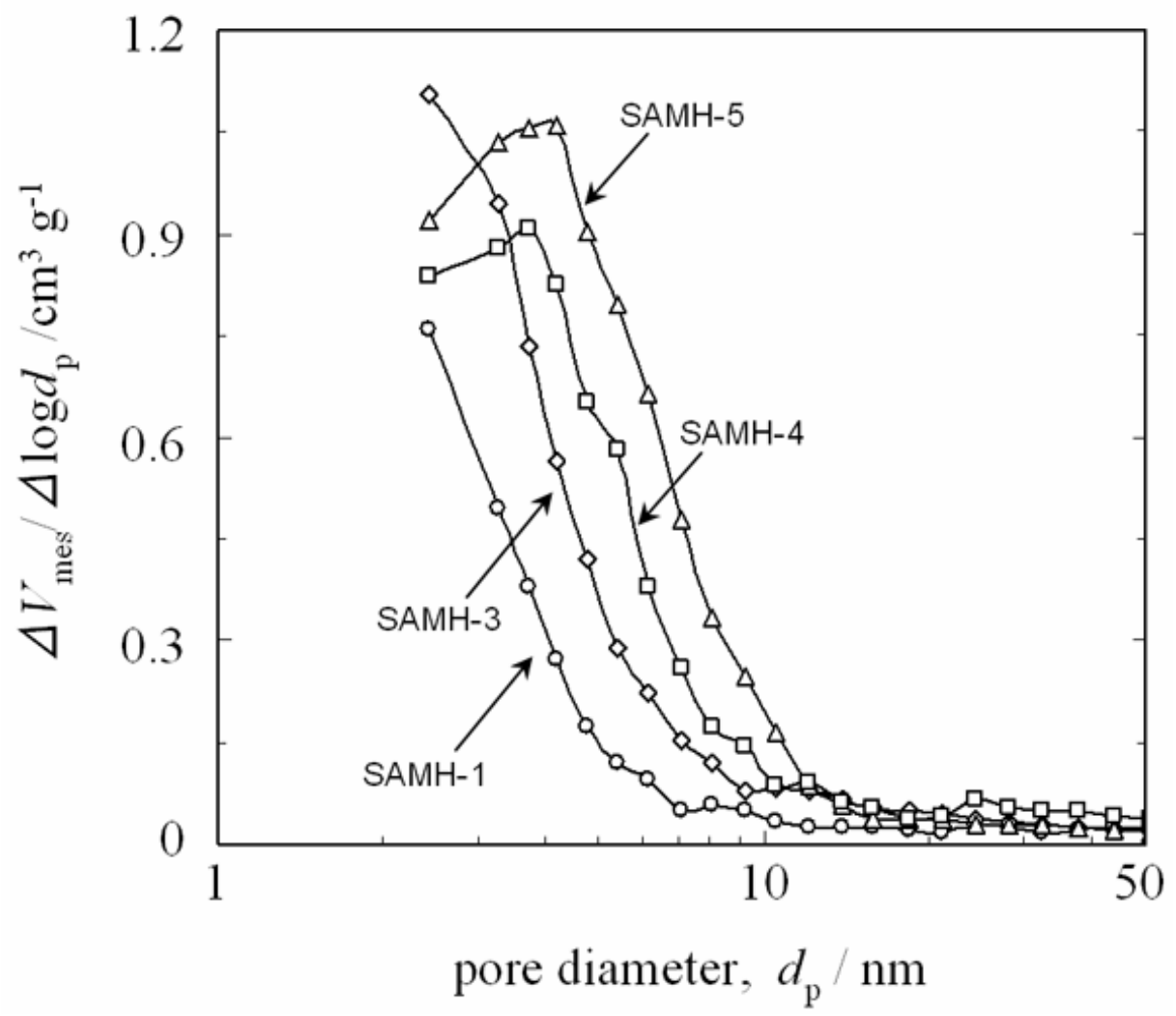

Mukai et al. Fig. 18 

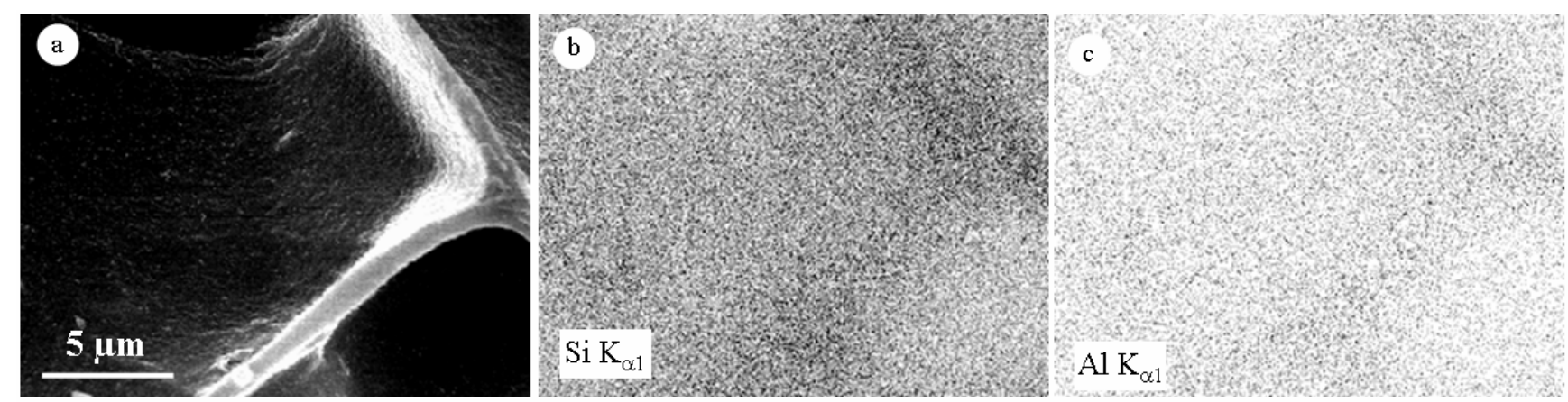

Mukai et al. Fig. 19 


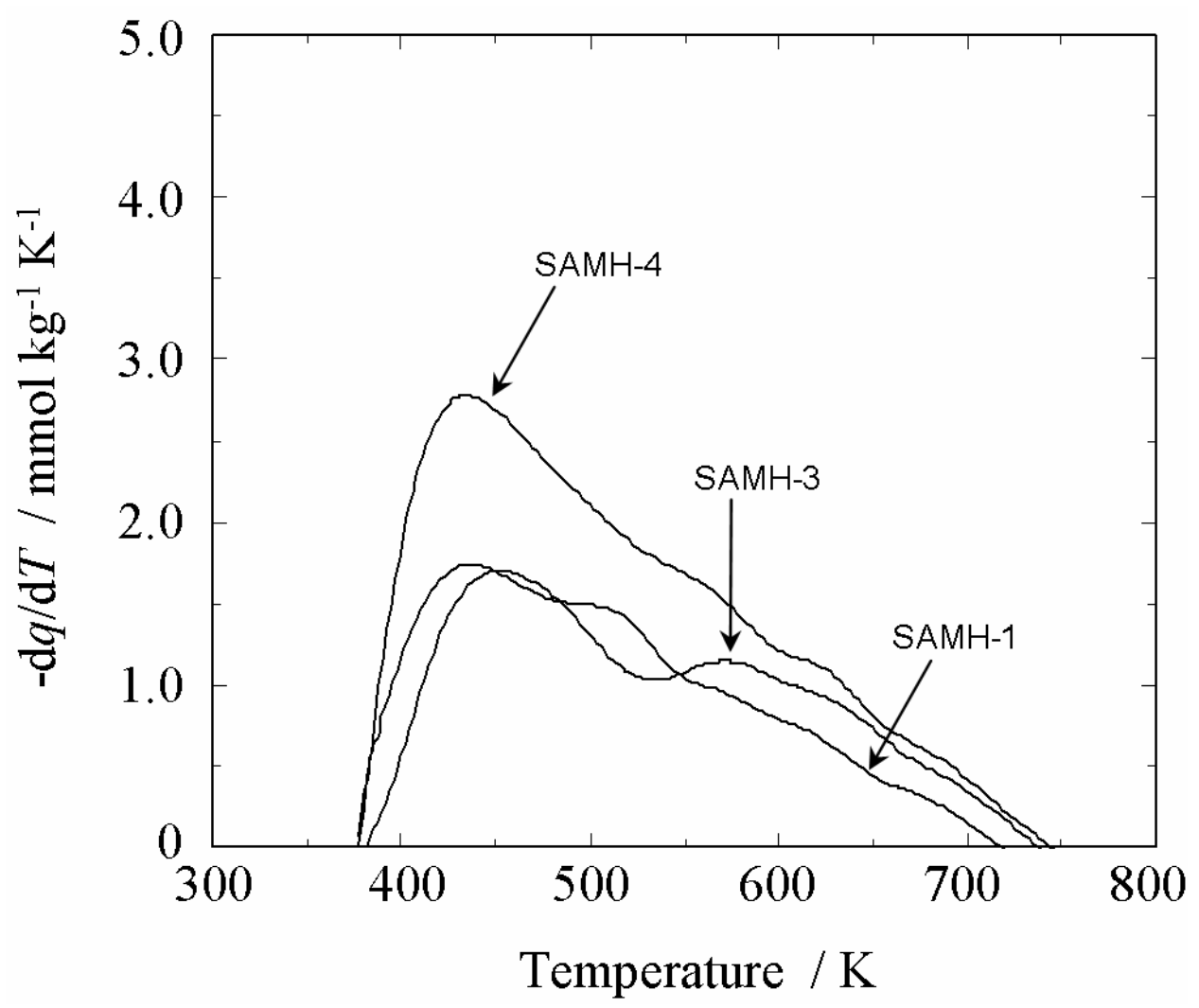

Mukai et al. Fig. 20 